Sharif University of Technology
Scientia Iranica
Transactions A: Civil Engineering
IRAENTIA
http://scientiairanica.sharif.edu

Research Note

\title{
Online nonlinear structural damage detection using Hilbert Huang transform and artificial neural networks
}

\author{
S.M. Vazirizade ${ }^{a, *}$, A. Bakhshi ${ }^{\mathrm{a}}$, and O. Bahar ${ }^{\mathrm{b}}$ \\ a. Department of Civil Engineering, Sharif University of Technology, Tehran, Iran. \\ b. International Institute of Earthquake Engineering and Seismology, Tehran, Iran. \\ Received 3 April 2018; received in revised form 12 December 2018; accepted 3 March 2019
}

KEYWORDS
Online damage
detection;
Structural health
monitoring;
Ensemble empirical
mode decomposition;
Moment-resisting steel
frame;
Artificial neural
network;
Hilbert
transformation.

\section{KEYWORDS}

Structural health

Ensemble empirical

mode decomposition;

frame;

Artificial neural

Hilbert

transformation

\begin{abstract}
In order to implement a damage detection strategy and assess the condition of a structure, Structural Health Monitoring (SHM) as a process plays a key role in structural reliability. This paper aims to present a methodology for online detection of damages that may occur during a strong ground excitation. In this regard, Empirical Mode Decomposition (EMD) is superseded by Ensemble Empirical Mode Decomposition (EEMD) in the Hilbert Huang Transformation (HHT). Although analogous with EMD, EEMD brings about more appropriate Intrinsic Mode Functions (IMFs). IMFs are employed to assess the first-mode frequency and mode shape. Afterwards, Artificial Neural Network (ANN) is applied to predict story acceleration based on previously measured values. Because ANN functions precisely, any congruency between predicted and measured accelerations indicates the onset of damage. Then, another ANN method is applied to estimate the stiffness matrix. Though the first-mode shape and frequency are calculated in advance, the process essentially requires an inverse problem to be solved in order to find stiffness matrix, which is done by ANN. This algorithm is implemented on moment-resisting steel frames, and the results show the reliability of the proposed methodology for online prediction of structural damage.
\end{abstract}

(C) 2019 Sharif University of Technology. All rights reserved.

\section{Introduction}

Civil structures in general and those subject to seismic excitation in particular are vulnerable to damage and deterioration during their service life. Inasmuch as damages to civil systems have led to unwanted major loss and casualty, they have gained the attention of the scientific community. The process of assessing the condition of a structure in order to detect any imperfec-

*. Corresponding author. Tel.: +15208095049

E-mail addresses: s.m.vazirizade@gmail.com (S.M.

Vazirizade); bakhshi@sharif.edu (A.Bakhshi);

omidbahar@iiees.ac.ir (O.Bahar)

doi: $10.24200 /$ sci.2019.50657.1808 tion is done by visual inspection traditionally and some conventional methods. These antediluvian methods are susceptible to becoming obsolete. Thus, new methods, that is, Non-Destructive Evaluation techniques (NDE), are designed [1]. Although such new methods as acoustic signals, electromagnetic, radiography, fiber optics, and so forth are not only more effective and convenient but also more economical, these damage detection methods are not global, but local. Therefore, they are effective only for small structures or structural members [2]. Global vibration-based techniques have been released recently in order to overcome this challenge. These methods contain Fourier transform, power spectrum, and spectrum analysis, to name a few [3]. As sensors and data acquisition systems have become more affordable during the preceding decades, 
the use of vibration data to find an effective strategy for the purpose of quantifying structural damage in engineering structures has gained more attention; even there are some studies that use this huge amount of data more efficiently [4]. A review of vibrationbased health monitoring methods can be found in [510]. The vibration-based methods, considering simply frequency, are worthwhile. Nonetheless, these methods discard time information. Additionally, most of the signals in the real world are not only nonlinear but also non-stationary concurrently, which require following new methods rather than conventional ones. In order to solve these problems, time-frequency methods are implemented, which present both frequency and time simultaneously. Furthermore, they can be used for nonstationary signals [9]. Hilbert Transform (HT) is a device in time-frequency domain for signal processing and is used for structural damage detection[11-13]. Wavelet Transform (WT) and Short Fast Furrier Transform (SFFT) are other tools for time-frequency analyses, and the former also provides variable-sized regions for windowing [14]. In doing so, higher frequency resolutions are brought, and a uniform resolution for all scales is provided [15]. However, WT and SFFT have limitations in Time-Frequency representation of non-stationary signals [16]. Huang et al. [17,18] firstly developed a new method-Hilbert Huang Transformation (HHT)-in order to analyze both nonlinear and non-stationary signals. This aforementioned method is composed of two portions, that is, Empirical Mode Decomposition (EMD) and HT. In fact, the first step, so-called EMD, is a procedure that provides some signals that stem from the main one. In other words, the mother signal is decomposed into Intrinsic Mode Functions (IMF). Since the EMD is based on the local characteristic time scale of the original data, this decomposition method is adaptive and highly efficient. The HHT method at a galloping rate has been employed in many scientific and engineering disciplines in general and structural health monitoring in particular to give new insights into the non-stationary and nonlinear signals [19]. Huang et al. [20] asserted that HHT is not only a more precise definition of particular events in time-frequency space than wavelet analysis, but also more physically meaningful interpretation of the underlying dynamic processes, too. Vincent et al. [21] compared the EMD method with wavelet analysis for structural damage detection. The structure, which is a simple 3-DOF system, was monitored during 20 seconds. The stiffness of the first story decreased, and the instantaneous frequencies were calculated from the first five IMFs. It was concluded that both the EMD and wavelet methods were effective in detecting the damage; however, the EMD method seems to be more promising for quantifying the damage level. Many assorted methods have been proposed to improve HHT in decomposition portion, EMD, and HT portion. In this regard, by utilizing the benefits of the properties of white noise to distribute components with more proper scales, Ensemble EMD (EEMD) has been presented due to resolving the mode mixing problem in EMD [22,23]. Aied et al. [24] used EEMD to detect sudden stiffness changes in a bridge model. Furthermore, they argued that the application of EEMD seems to be more adaptive to nonlinear signal than that of wavelets. Moreover, they asserted that by using EEMD rather than EMD, the mode mixing problem diminishes significantly. It should be kept in mind that environmental conditions, such as temperature, might have significant effects; however, sudden changes in stiffness are always of importance [25]. Nagarajaiah and Basu [26] conducted a very comprehensive research on the most common time-frequency techniques; first, they developed output-only modal identification as well as evaluated frequencies, damping ratios, and mode shapes of MDOF LTI and LTV systems. Then, besides using HT, they applied SFFT, EMD, and wavelet and discussed the performance of each one. However, they did not employ EEMD. Today, improvements in computers' processing power pave the way for more elaborate computations. Artificial intelligence, therefore, has become the focus of attention in recent decades. Artificial Neural Networks (ANNs) in terms of machine learning and cognitive science are learning models inspired by biological neural networks [27]. Suresh et al. [28] considered the flexural vibration in a cantilever beam. A neural network was trained by modal frequency parameters-calculated for various locations and crack depths. Furthermore, they investigated two widely used neural networks, namely the multi-layer perceptron network and the radial basis function network, and figured out that the latter was better than the former by virtue of performance and computational time. The effects of three different learning rate algorithms-the Dynamic Steepest Descent (DSD) algorithm, the Fuzzy Steepest Descent (FSD) algorithm, and the Tunable Steepest Descent (TSD) algorithm-on the neural network training were studied. Fang et al. [29] studied the Back-Propagation Neural Network (BPNN) and used Frequency Response Functions (FRFs) as its input data in order to assess damage conditions of a cantilever beam. Eventually, they asserted that this new approach is highly accurate in predicting damage location and severity. $\mathrm{Xu}$ et al. [30] presented a method for the direct identification of structural parameters based on neural networks. They used two back-propagation neural networks the first of which, emulator, was employed to predict its dynamic response with sufficient accuracy using timedomain dynamic responses. Then, the difference between structure response and neural network prediction was assessed with Root Mean Square (RMS). The 
second neural network, called parametric evaluation neural network, was proposed to identify the structural parameters. Saadat et al. [31] proposed a method called the Intelligent Parameter Varying (IPV) that uses radial basis function networks to estimate the constitutive characteristics of inelastic and hysteretic restoring forces and showed the effectiveness of this method. Bandara et al. [32] utilized a neural network for the actual damage localization and quantification based on FRF. Their network inputs were damage patterns of different damage cases associated with FRF, and outputs were either the damage locations or severities. By having a sufficient amount of data, a deep learning approach can be applied to both global and local health condition assessment of structures [33]. Entezami et al. [34] used an unsupervised learning method to extract features required for structural health monitoring. A quality literature review about different methods for feature extraction can be found in [35]. In this study, a methodology was proposed to detect damage in nonlinear moment-resisting steel frames. In fact, this method discerns the changes in structure stiffness as well as the onset of damage, predicts the location of damage, and measures the severity of damage by implementing EEMD, HT, and ANNs. Primarily, the first-mode frequency and mode shape are calculated by using EEMD and HT process. Afterward, by using two ANNs, the location and severity of damages are determined.

\section{Signal processing procedures}

\subsection{Hilbert Transform (HT)}

HT has proved useful to compute instantaneous frequency. By doing so, complex conjugate $y(t)$ of any real-valued function can be calculated. HT of a signal $x(t)$ is defined by:

$$
H[x(t)]=y(t)=\frac{1}{\pi} P V \int_{-\infty}^{+\infty} \frac{x(t)}{t-\tau} \mathrm{d} \tau,
$$

where $t$ is the time variable, and $P V$ indicates the principal value of the singular integral. With the HT, the analytic signal, $z(t)$, is obtained as follows:

$$
\begin{aligned}
& z(t)=x(t)+i y(t)=a(t) e^{i \theta(t)}, \\
& a(t)=\sqrt{x^{2}(t)+y^{2}(t)}, \\
& \theta(t)=\arctan \left(\frac{y}{x}\right),
\end{aligned}
$$

where $a(t)$ and $\theta(t)$ are the instantaneous amplitude and phase function, respectively. Instantaneous frequency is derived from the derivative of the phase function [36]:

$$
f(t)=\frac{1}{2 \pi} \frac{\mathrm{d} \theta(t)}{\mathrm{d} t} .
$$

In spite of the fact that HT has proved useful, one encounters difficulties for achieving physically meaningful instantaneous frequencies by applying HT. There are some conditions and theories that express these shortcomings, e.g., Bedrosian and Nuttall theorems, to name a few. In this regard, many assorted methods, such as Normalized Amplitude Hilbert Transform (NAHT) method and enhanced Hilbert Huang transform, have been proposed $[37,38]$. Reducing the signal into its IMFs has improved the chance of getting a meaningful instantaneous frequency. Thus, EMD is a procedure that decomposes a signal into its IMFs.

\subsection{Empirical Mode Decomposition (EMD)}

In 1996, EMD was proposed by Huang et al. [18] for nonlinear and non-stationary data. As mentioned earlier, EMD is a procedure that decomposes a signal into its IMFs. An IMF is defined as a function to satisfy two conditions:

1. The number of extrema and the number of zero crossings must either equal or differ at most by one in the whole data;

2. At any given point, the mean value of the upper envelope, defined by the local maxima, and the lower envelope, defined by the local minima, is zero.

To this end, amplitude and frequency of IMFs can be non-constant and changeable. In other words, amplitude and frequency are variable as a function of time; in doing so, HT becomes a helpful transformation.

For such a signal as $x(t)$, the EMD procedure can be summarized in six steps as follows:

1. Determining all of the local extrema, that is, maxima and minima;

2. Considering cubic spline passing all the local maxima as the upper envelope, $e_{\max }(t)$, and an analogous procedure for the local minima to achieve the lower envelope, $e_{\min }(t)$;

3. Computing the mean value of upper envelope and lower one:

$$
m_{i, j}=\left[e_{\min }(t)+e_{\max }(t)\right] / 2,
$$

where $i$ and $j$ indices indicate the number of associated IMFs and iterations, respectively. For the first IMF, $i$ equals 1 ;

4. Subtracting $m_{i, j}$ from the initial signal to find the first component, $h_{i, j}(t)$ :

$$
\begin{aligned}
& \text { if } j=1: h_{i, j}=x-m_{i, j}, \\
& \text { if } j \neq 1: h_{i, j}=h_{i, j-1}-m_{i, j} .
\end{aligned}
$$


5. Repeating steps 1 to 4 on $h_{i, j}(t) k$ times to obtain the next IMF, $C_{i}(t)$ :

$$
C_{i}=h_{i, k}
$$

6. Calculating $r$ and repeating steps 1 to 5 to extract the remaining IMFs, $r_{i+1}=x-C_{i}$, and a residue, $r_{n+1}(t)$.

Finally, by using these steps, $x(t)$ is decomposed to $n$-separate IMFs and a residue, $r_{n+1}(t)$. The original signal can be reconstructed by the sum of intrinsic modes and the residue:

$$
x=\sum_{i=1}^{n} C_{i}+r_{n+1} .
$$

Among the IMFs, high-frequency content is removed gradually from $C_{1}(t)$ to $C_{n}(t)$, resulting in the latter signals with lower frequency content.

\subsection{Ensemble Empirical Mode Decomposition (EEMD)}

Wu and Huang presented EEMD [22,23] to solve the mode mixing problem by adding a white noise to the original signal. Mode mixing, which is the consequence of signal intermittency, causes the existence of disparate scales in an IMF or the presence of a similar scale in different IMFs. Consequently, this drawback makes IMFs physically meaningless, and it is crucial to get rid of it. Therefore, EEMD is implemented for not only surmounting mode mixing problem but also reducing the sensitivity of a signal to noise pollution, as shown in this study. The statistical characteristics of white noise with an ensemble of trials bring about improvement in the scale separation problem. To put it differently, the addition of noise to EMD and iteration leads to a better sifting process. It should be mentioned that though the IMFs are polluted by noise in each trial, these effects are canceled out by utilizing ensemble mean. Wang et al. [39] compared the applications of EMD and EEMD on time-frequency analysis of seismic signals. They found that this new approach was remarkably capable of solving the mode mixing problem. They demonstrated this assertion by applying an example and revealed the ability of EEMD to decompose the signal into different IMFs and analyze the time-frequency distribution of the seismic signal.

EEMD is summarized within the following steps:

1. Adding white noise $w$ to the initial signal, $x(t)$, for the first try $k=1$ :

$$
x_{1}=x+w_{l} \text {. }
$$

2. Applying steps 1 to 5 of the EMD procedure to decompose signal $x_{1}(t)$ to its IMF.

3. Repeating steps (1) to (2) with different white noises for each try.
4. Averaging corresponding IMF calculated by each trial to obtain $C$ :

$$
C_{i}=\lim _{n \rightarrow \infty} \frac{1}{n} \sum_{l=1}^{n} C_{i, l},
$$

where $n$ is the number of ensembles.

5. Subtracting $C_{i}(t)$ from the initial signal and repeating these steps for finding the remaining IMFs.

Finally, the main signal can be reproduced as in the following equation:

$$
x=\sum_{i=1}^{n} C_{i}+r_{n+1} .
$$

The main advantage of EEMD over EMD is that the former is less sensitive to noise than to EMD. In other words, the IMFs extracted by EEMD are more analogous with each other than with those of EMD while the uniformity among the decomposed signals is of considerable importance for extracting the mode shapes.

\section{Damage detection procedure}

In this section, the rewarding process of finding severity and location of damage, which is a combination of signal processing and artificial intelligence, is proposed. Figure 1 plots the schematic process of the proposed method for a typical 3-story moment-resisting frame. In the following steps, the procedure is closely examined.

The first step is recording the response of the structure using installed sensors. It should be mentioned that because of the earthquake recorded as an excitation at the base level of the structure as well as the damping effect on the structure, the corresponding response is non-stationary. Another point deserving attention is that the procedure is output-only, and in order to underline this fact, there are not any installed sensors on the base although it could be. As mentioned earlier, the first exquisite step is recording the structural response in each story. These signals are considered as input data for EEMD, and the extracted results are IMFs. Then, HT is applied to IMFs to find instantaneous frequencies and amplitudes. It should be mentioned that each story signal is satisfied in order to evaluate the first-mode frequency, and the result can be double-checked by the others. Additionally, in most cases, the structural response is more obvious in higher elevations; thus, the last story response is considered as the main signal for finding the dominant frequency. It is further shown that the results are not significantly sensitive to the first-mode dominant frequency. In the next step, thanks to more uniform IMFs by virtue of EEMD, each story' IMFs containing the first-mode 


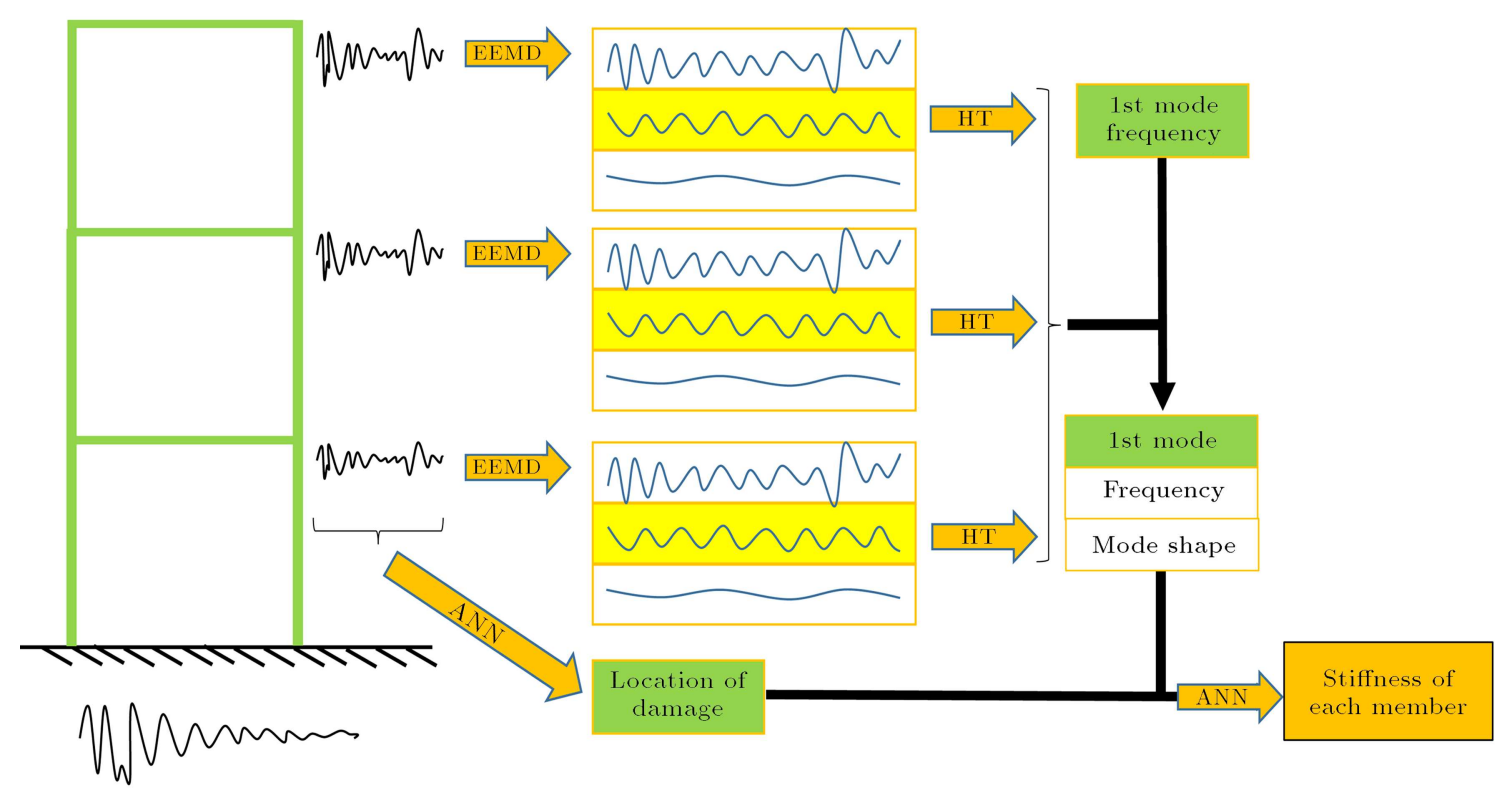

Figure 1. Schematic view of the proposed method.

frequency is normalized in relation to the those of the last story. In doing so, the first-mode shape is at hand.

Then, a Radial Basis Function Network (RBFN) is employed in order to predict the response of each story. The task of this network is to forecast the acceleration of each story using its acceleration in previous moments and other stories in the current and previous moments. To put it differently, by using early second responses, as long as the excitation is not destructive, or the former excitation is not strong enough to damage the structure, this network is trained and ready to predict the behavior of the structure. As mentioned earlier, any differences between the predicted and measured values signify changes in structural elements on the grounds that RBFNs are remarkably powerful to predict acceleration response precisely. This step is extremely worthwhile to locate the damage and facilitate the procedure for the next step.

The last step is applying another network to measure the severity of damage. Although the aforementioned steps are a non-model based method, the intact and undamaged structure is required to be compared with the damaged one in this step. Furthermore, this network needs some data to train. In other words, if the relationship between the first-mode shape and frequency and its corresponding parameters of the associated structure is available, the structural parameters can be identified based on the first-mode shape and frequency; however, a formidable challenge is to establish a mathematical model for mapping from the mode shape and frequency to the structural parameters. Although the Eigen values and Eigen vectors are simply at hand with known stiffness matrix and, by doing so, mode shapes and frequencies are calculated, it is a formidable task to reach a general solution to assess the stiffness matrix by the firstmode shape and frequency. Therefore, the application of optimization algorithms can be helpful in solving this inverse problem. These optimization algorithms include Genetic Algorithm (GA), Swarm Intelligence (SI), Particle Swarm Optimization (PSO), Multiparticle Swarm Coevolution Optimization (MPSCO), and Improved Multiparticle Swarm Coevolution Optimization (IMPSCO) employed by Jiang et al. [40] to localize and quantify the structural damage in comparison with GA, etc. Friswell et al. [41] applied the genetic algorithm and vibration data to the problem of damage detection. In this study, an ANN is applied on the grounds that the neural network has the ability to approximate arbitrary continuous function and mapping, too. Therefore, it stands to reason that the mathematical model is supplanted by a network that can be considered as an optimization problem with the severity of the damages as its variables.

\section{Modeling and analysis}

The first structure is a three-story steel momentresisting frame with story height and spans of $3 \mathrm{~m}$ and $4 \mathrm{~m}$, respectively, and its specifications are provided in Table 1. The open-source finite element program, OpenSees, is used for nonlinear dynamic analysis. The utilized material is Steel01, which is a bilinear model. By using 0\% strain hardening, it is transformed into elastic-perfectly plastic model, in which all of these parameters are summarized in Table 2 , where $F_{y}, E$, and $\delta_{y}$ represent yielding stress, modules of elasticity, and yielding strain. This structure has Rayleigh's proportional damping with both the first and the third modal damping ratios of 0.05 . The scaled Northridge 
Table 1. Specifications of the three-story frame.

\begin{tabular}{cccc}
\hline Story & Mass (kg) & $\begin{array}{c}\text { Stiffness of beam } \\
\text { section (MPa) }\end{array}$ & $\begin{array}{c}\text { Stiffness of column } \\
\text { section (MPa) }\end{array}$ \\
\hline All & 8000 & 8.4164062 & 17.45226 \\
\hline
\end{tabular}

Table 2. The material properties used for modeling the structure.

\begin{tabular}{ccccc}
\hline Material no. & $\begin{array}{c}\boldsymbol{F}_{\boldsymbol{y}} \\
(\mathbf{M P a})\end{array}$ & $\begin{array}{c}\boldsymbol{E} \text { elastic } \\
(\mathbf{M P a})\end{array}$ & $\begin{array}{c}\boldsymbol{E} \text { plastic } \\
(\mathbf{M P a})\end{array}$ & $\boldsymbol{\delta}_{\boldsymbol{y}}$ \\
\hline 1 & 200 & $2 \times 10^{5}$ & 0 & 0.001 \\
\hline
\end{tabular}

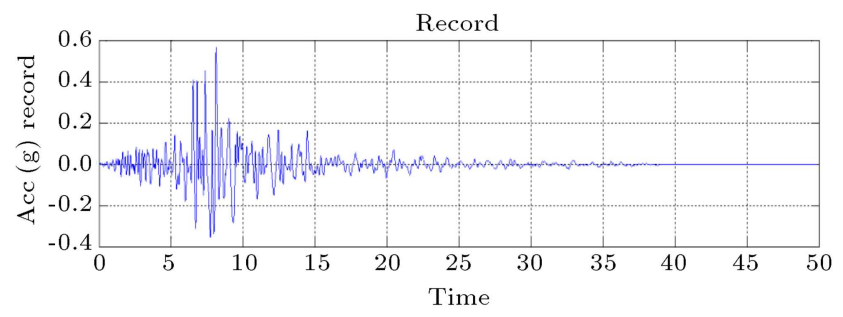

Figure 2. Scaled Northridge ground motion acceleration record.

earthquake record (which is multiplied by two in order to push the structure into plastic range) is applied to the structure and the ground motion, as shown in Figure 2.

Apart from the mentioned points, in order to simulate damage in columns, each return to the elastic range after undergoing plastic behavior reduces column stiffness to $91 \%$ of its previous stiffness, which is an assumed value that indicates those changes less than $15 \%$. This reduction is imposed on modulus of elasticity, and in order to simulate sudden damages and cracks in the elements, it appears after the occurrence of plastic region. In this regard, it should be mentioned that although both beams and columns tolerate nonlinear behavior during a seismic excitation, only columns are susceptible to degradation. These changes in structural element stiffness are demonstrated in Figure 3 where the second and third story columns are not shown, because they remain in the elastic range.

In this study, two salient features of EEMD are considered: the noise level and ensemble number. As the names imply, the amplitude of noise is determined according to the standard deviation of original data in each step and the number of iterations, respectively. In spite of the fact that a few hundred ensembles bring about appropriate results, there is no definite value for the noise level [23]. Figure 4 shows IMFs using EMD of the base and the first to the third story from left to right. In fact, if the noise level is 0 and ensemble number is 1, EEMD changes into EMD, and Figure 5 displays its corresponding HT. Even though IMFs are improved by increasing the ensemble number, 5000 is enough in number because no
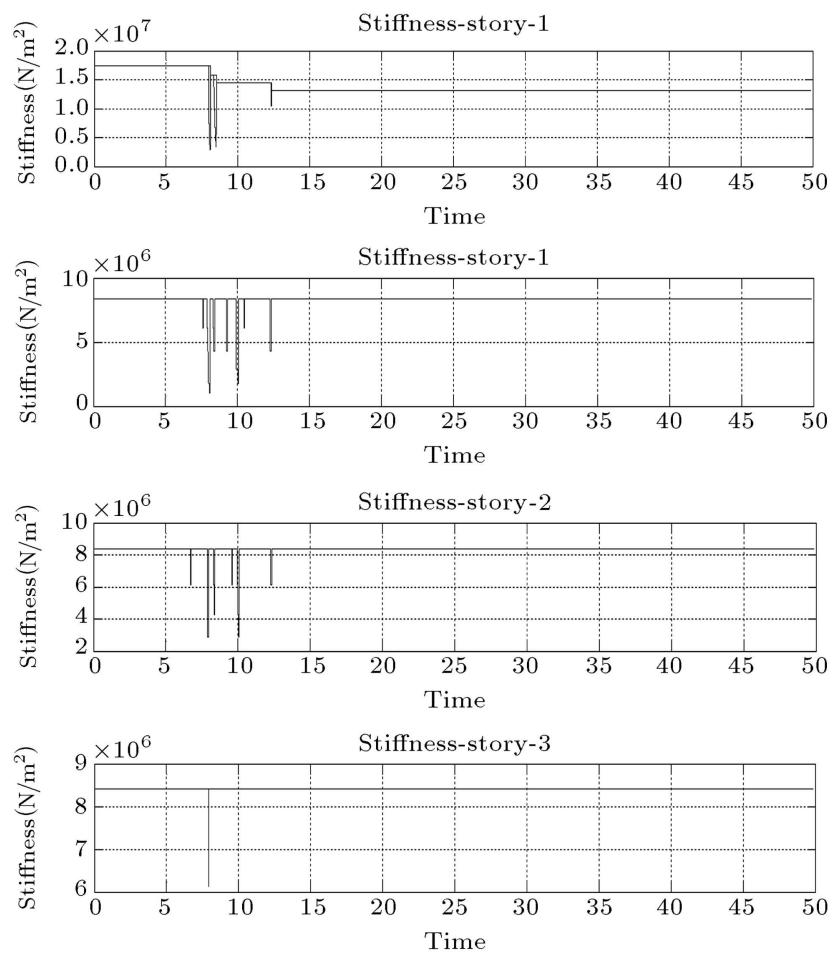

Figure 3. Changes in beams and first story columns stiffness.

significant difference is observed after this number; the values more than this number would merely increase the computational cost. Furthermore, the noise level, which is less than 0.5 , is not a completely acceptable remedy. Therefore, it is better to use values more than 0.5. Figure 6 shows HT of the seventh and eighth IMFs with the noise level of 1 and ensemble number of 5000 . There is a histogram close to every HT, which is a new method. The dominant frequency is assessed by these histograms rather than the conventional marginal distribution. Instantaneous frequency of the structure changes due to the susceptibility of the structure to plastic deformation and instant changes in stiffness matrix. Thus, it is probable for HT to provide large values that affect the marginal distribution. On the contrary, constructing a histogram is based on the number of values that fall into each interval; therefore, 

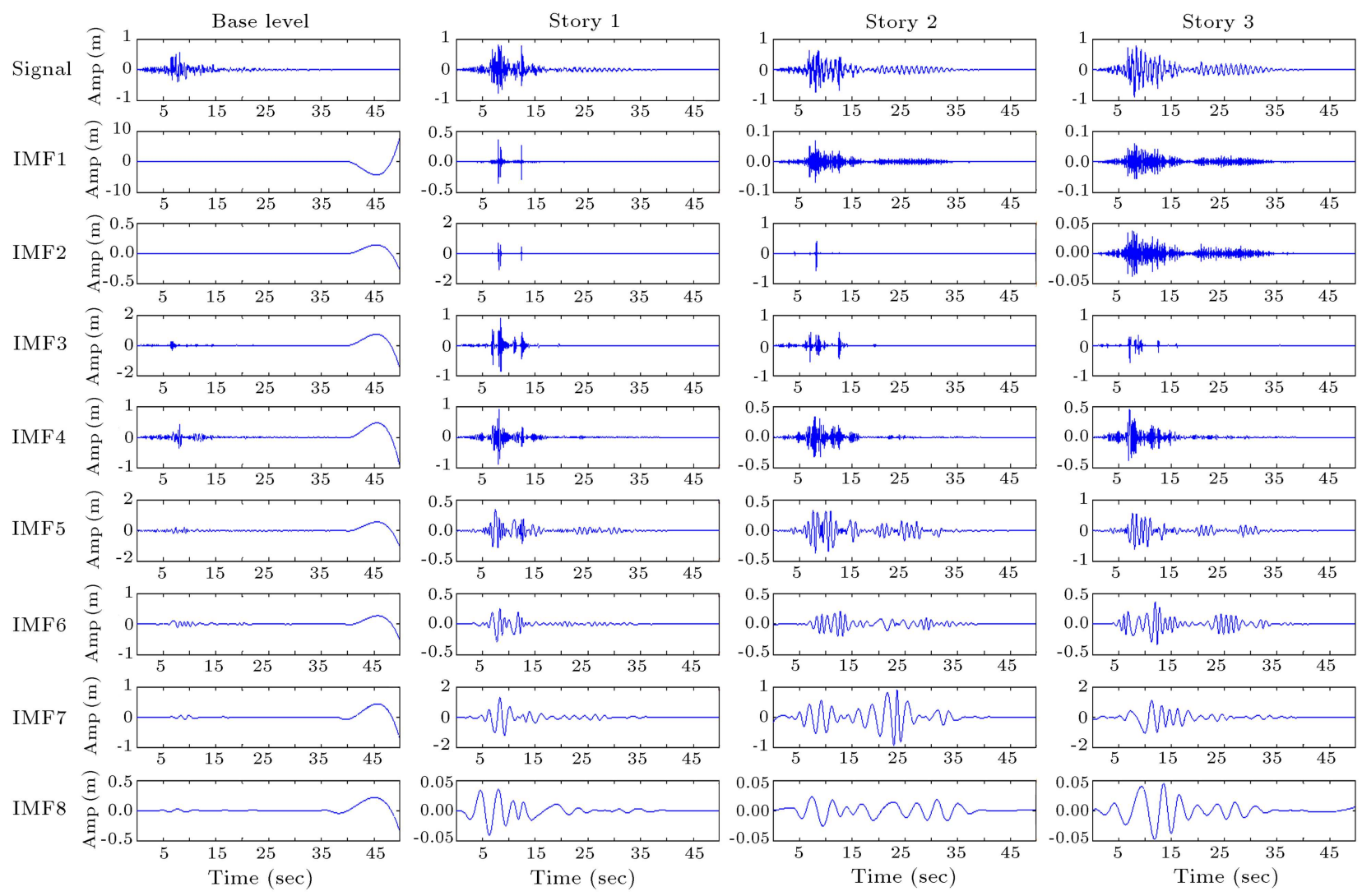

Figure 4. The ground motion acceleration and structure acceleration response IMFs with noise level of 0 and ensemble number of 1 .

Table 3. Comparsion of FEM results and proposed method results.

\begin{tabular}{ccccc}
\hline & $\begin{array}{c}\text { Frequency 1st } \\
\text { mode }(\mathbf{H z})\end{array}$ & \multicolumn{3}{c}{ Mode shapes } \\
\cline { 3 - 5 } & & 1st story & 2nd story & 3rd story \\
\hline Calculated by FEM model & 1.0696 & 0.2964 & 0.7037 & 1 \\
Calculated by EEMD and Hilbert & 1.07 & 0.2978 & 0.7055 & 1 \\
Percentage of error & $0.5^{*}$ & 0.5 & 0.3 & 0 \\
\hline
\end{tabular}

${ }^{*}$ Center of $[1.0651 .075)$ is 1.07 . Therefore, maximum error is $(1.075-1.0696) / 1.0696=0.005$.

effects of inordinate amplitude in estimating dominant frequency are squandered. In Figure 6, apart from the first column relating to ground motion, the seventh and eighth IMFs exhibit the lowest meaningful dominant frequency that emanates from the first-mode vibration. Furthermore, Figure 7 presents normalized total amplitude of the seventh and eighth IMFs for each story relative to those of the third story for different values of the noise level and ensemble number. According to this figure, as mentioned earlier, the noise level at or below 0.5 does not produce decent results. Meanwhile, when the noise level is 1 or 2 , the results closely resemble each other. Moreover, Figure 7 depicts the mentioned normalized amplitude according to the ensemble number. In this regard, if the ensemble number is 5000 or 10000 , normalized amplitudes in each column are the same.

According to Figure 7, the longest duration in which variance of values in normalized amplitudes reaches a stable and minimum level is considered as the normalized mode shape; therefore, for example, when the ensemble number is 5000 and noise level is 1 , this timespan spans from 6 to 36 seconds. Table 3 compares the first-mode shape and frequency calculated by Finite Element Method (FEM) with the proposed method briefly.

The next step is employing two networks, discussed before. The schematic architecture of the first network is illustrated in Figure 8. In fact, this network needs a vector with $m \times n+n-1$ members, where $m$ 

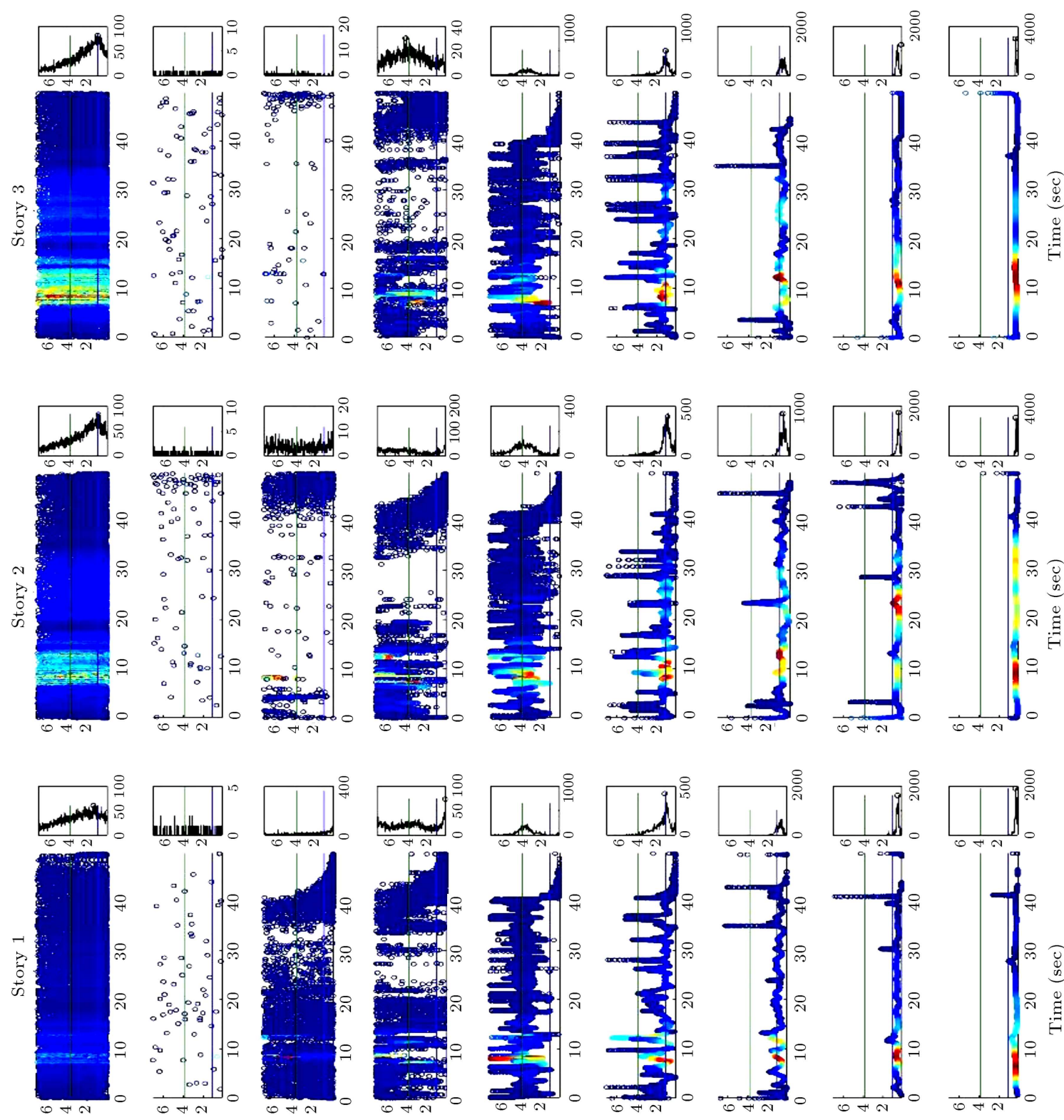

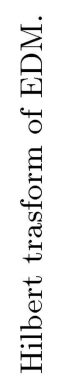
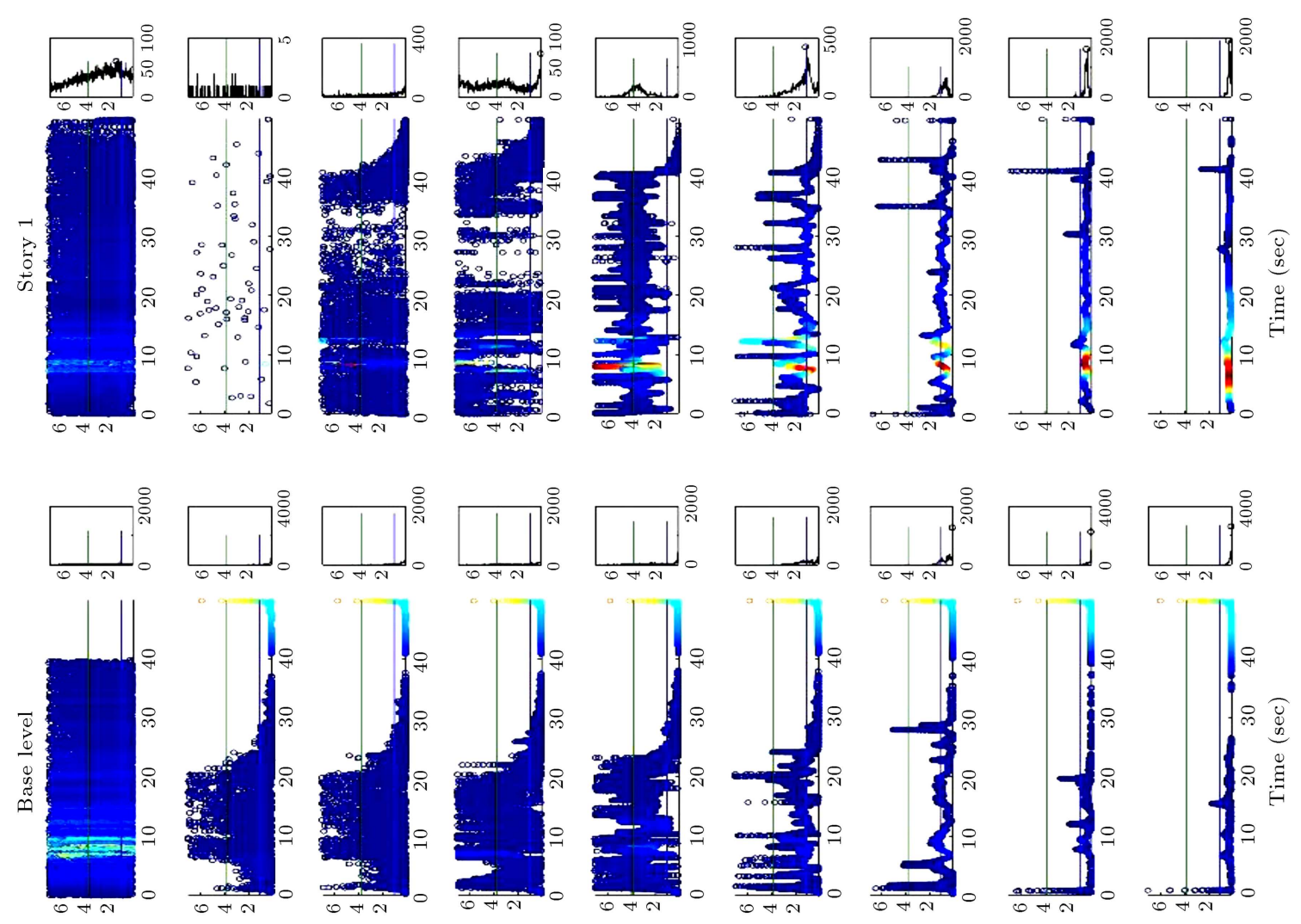

$\left(\mathrm{z}_{\mathrm{H}}\right)$ bə.І변

$\left(\mathrm{z}_{\mathrm{H}}\right)$ bə.І뱁

$\left(\mathrm{z}_{\mathrm{H}}\right)$ bə.⿸丆𠃋

$\left(\mathrm{z}_{\mathrm{H}}\right)$ bәл변

$\left(\mathrm{z}_{\mathrm{H}}\right)$ bə.

$\left(\mathrm{z}_{\mathrm{H}}\right)$ bәлн

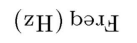

$\left(\mathrm{z}_{\mathrm{H}}\right)$ bə.⿸丆𠃋⿺乚丶

$\left(\mathrm{z}_{\mathrm{H}}\right)$ bə. $\mathrm{x}_{\mathrm{H}}$
高
产
毵
旁
童
墨
量
童
$\sum_{=}^{\infty}$ 


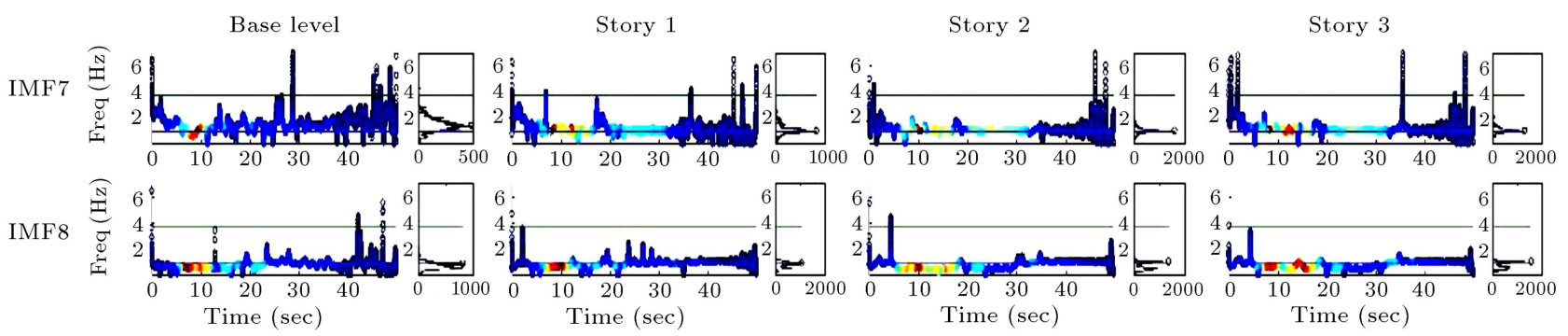

Figure 6. Hilbert transform of the IMFs 7th and 8th with noise level of 1 and ensemble number of 5000 .
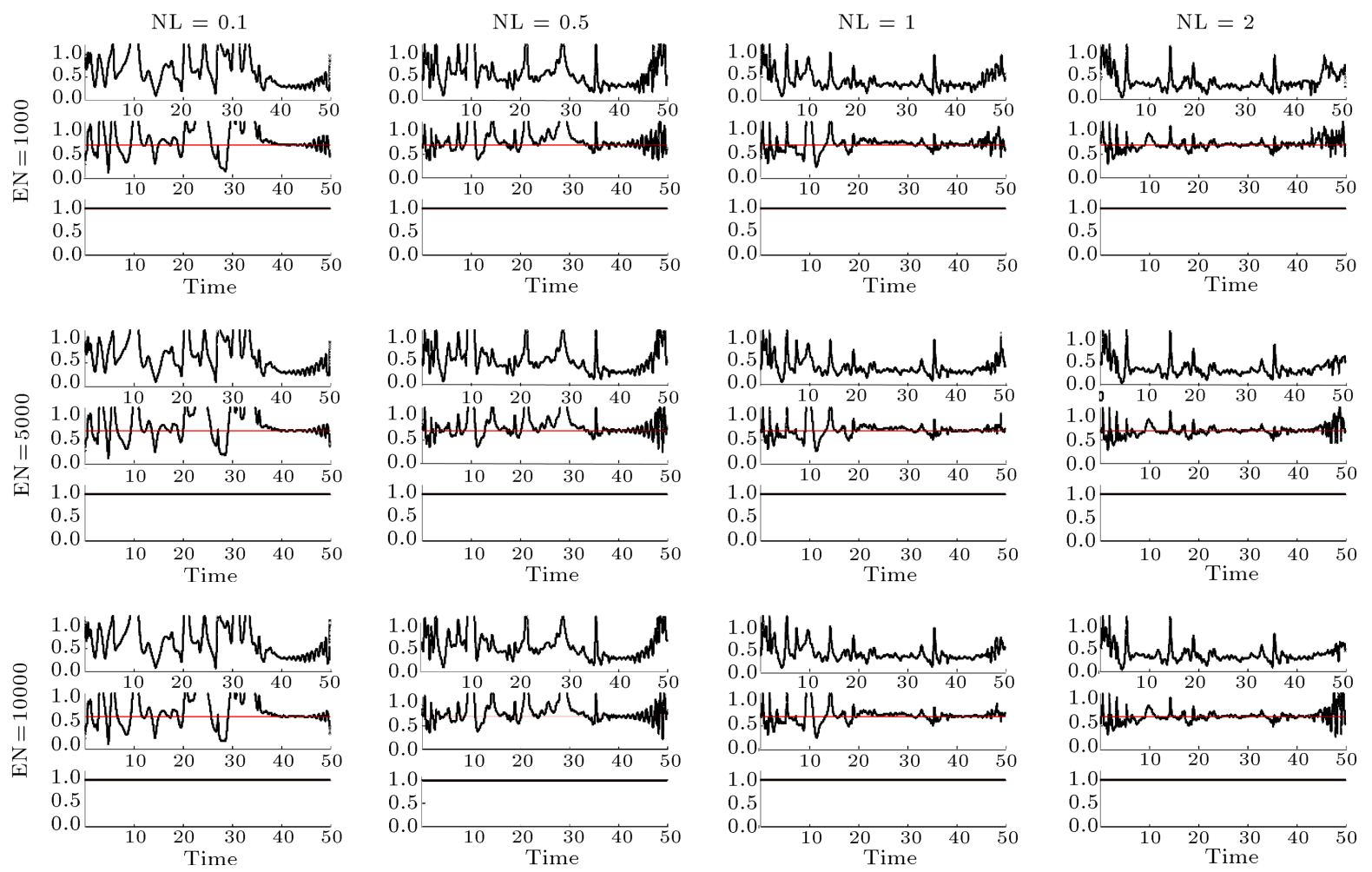

Figure 7. Normalized total amplitude of the IMFs 7th and 8th for each story relative to story 3.

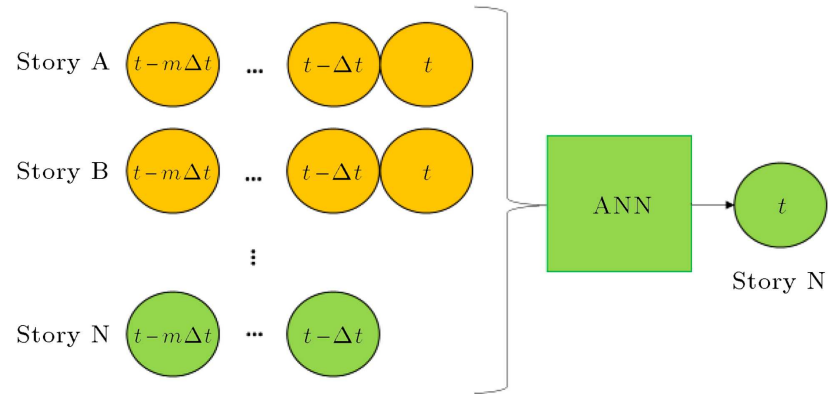

Figure 8. Overview of emulator artificial neural network.

is the required previous moment, and $n$ is the number of stories. Consequently, $m \times n$ and $n-1$ define the total number of input data based on previous moments and current moment, respectively. In this study, $n$ is 3 due to the structure stories and $m$ is 4 , although many other values have been tested. This network is trained for each story (for this 3-story frame, 3 networks are required and trained. Their structures are similar, yet are different only in input and output data), and the predicted acceleration responses are shown in Figure 9. Another subtle point deserving to be mentioned is that in order to predict the acceleration response in the $k$ th story, previous moment acceleration responses for the $(k-1)$ th, $k$ th, and $(k+1)$ th stories, as well as current moment acceleration responses for the $(k-1)$ th and $(k+1)$ th stories, are sufficient. In other words, the response data of lower and upper stories are enough; however, the alluded measures have been taken due to the lack of a sensor at the base level of this frame. Therefore, using a sensor at the base level not only improves the performance of the network but also diminishes the input data. 

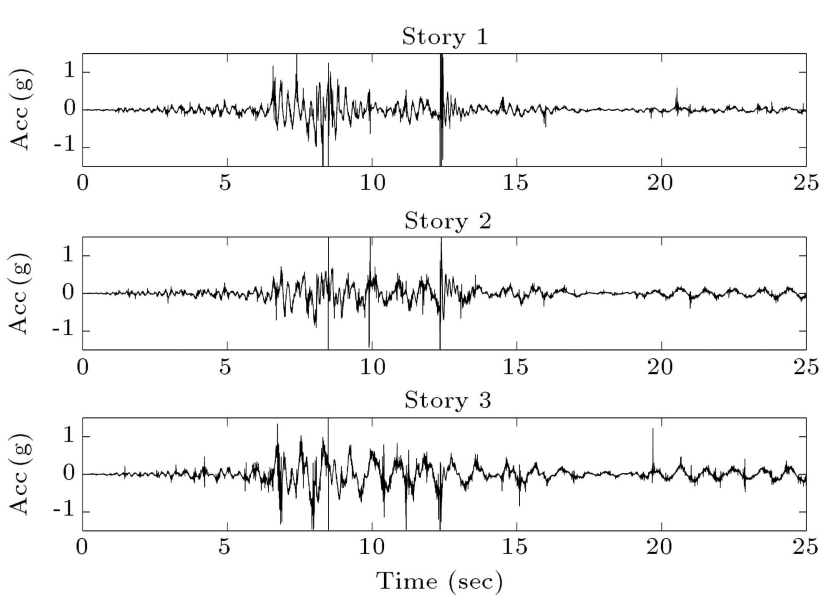

Figure 9. The predicted acceleration response of the structure.
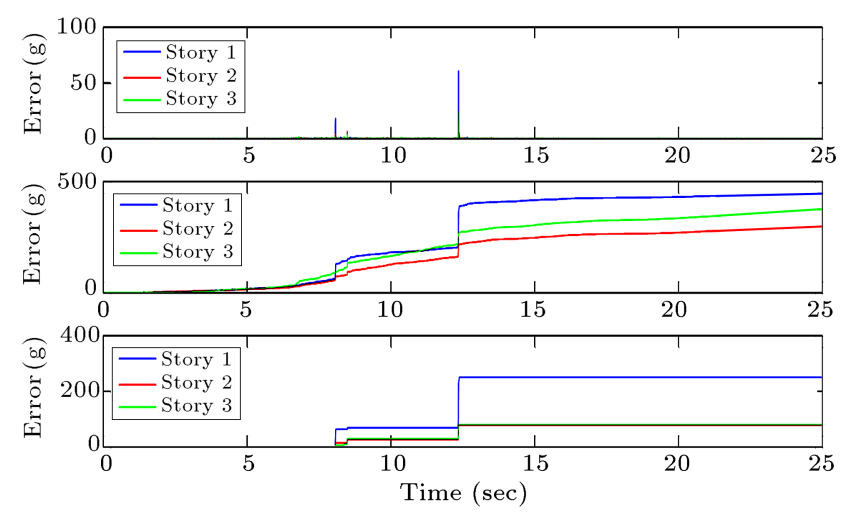

Figure 10. The difference between structure response and neural network prediction.

Figure 10 presents the difference between structure responses and neural network predictions, that is, absolute difference, cumulative difference (showing a gentle slope owing to the noise added to input and output data), and modified cumulative difference (eliminating the gentle slope) in rows one to three, respectively. The big jumps in the first story error reveal changes in structure behavior, and the vicinity of two other stories' errors to those big jumps shows the presence of anomaly in the predicted data, stemming from one common source, i.e., the first story.

After locating damages and determining the firstmode parameters, the final step is the second network, as shown in Figure 11. This network is trained by 100 sets of training patterns, associated with the performance of the previous network. The summarized results are presented in Table 4 . In addition, Figure 12

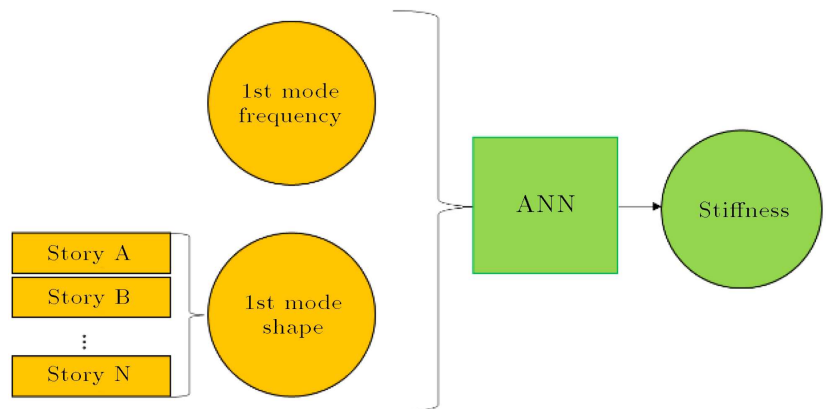

Figure 11. Overview of the second artificial neural network.

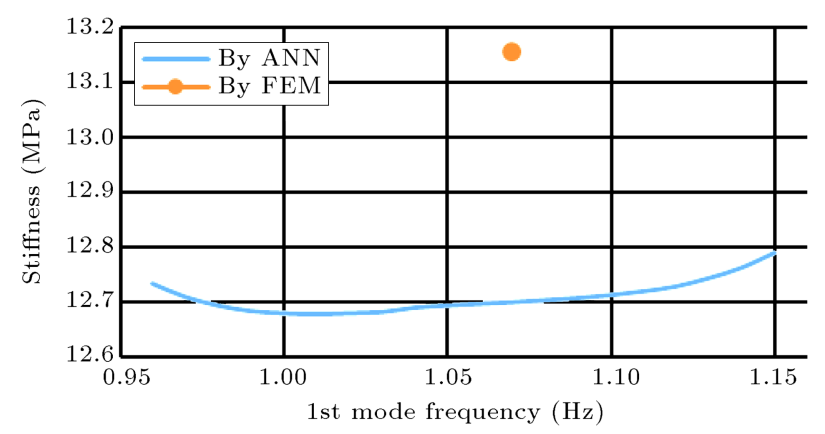

Figure 12. Sensitivity of the proposed algorithm to the first-mode dominant frequency.

shows the sensitivity of the proposed algorithm to the first-mode dominant frequency. Indeed, because of using histogram rather than marginal distribution, the first-mode frequency is the interval center; nevertheless, this figure sheds light on the fact that the length of bins is not the determining factor.

Another example is a 6-story frame subjected to Fruili earthquake record at the base level. The procedure is the same as the previous structure; however, there are two damaged stories-the first and sixth stories-the stiffness of which has actually decreased. Figure 13 shows the amplitude of IMF number 7 for each story relative to the sixth story and elaborates that EEMD is a useful device to assess the first-mode shape.

Furthermore, as mentioned earlier, any changes in stiffness can be detected by the difference between structure responses and neural network predictions.

Figure 14 depicts absolute difference, cumulative difference, and modified cumulative difference after eliminating the gentle slope. The first and sixth stories represent relatively more significant error than those of the other stories.

Table 4. Comparsion of FEM results and the proposed method results.

\begin{tabular}{cccc}
\hline Damaged story & $\begin{array}{c}\text { Evaluated stiffness } \\
(\mathbf{M P a})\end{array}$ & $\begin{array}{c}\text { Calculated by FEM } \\
\text { (MPa) }\end{array}$ & Percentage of error \\
\hline$\# 1$ & 12.70 & 13.15 & 3.4 \\
\hline
\end{tabular}


Table 5. Comparsion of FEM results and proposed method results.

\begin{tabular}{|c|c|c|c|c|c|c|c|}
\hline & \multicolumn{7}{|l|}{ Frequency } \\
\hline & \multirow{2}{*}{$\begin{array}{c}\text { 1st mode } \\
(\mathrm{Hz})\end{array}$} & \multicolumn{6}{|c|}{ Mode shapes } \\
\hline & & 1st story & 2nd story & 3rd story & 4th story & 5 th story & 6 th story \\
\hline Calculated by FEM model & 0.8326 & 0.1302 & 0.3412 & 0.5525 & 0.7503 & 0.8968 & 1 \\
\hline Calculated by EEMD and Hilbert & 0.830 & 0.1303 & 0.3366 & 0.5447 & 0.7493 & 0.9057 & 1 \\
\hline Percentage of error & $0.9^{*}$ & 0.1 & 1.3 & 1.4 & 0.1 & 1.0 & 0 \\
\hline
\end{tabular}

*Center of $[0.8250 .835)$ is 0.83 . Therefore, the maximum error is $(0.8326-0.825) / 0.8326=0.00)$.

Table 6. Comparsion of FEM results and the proposed method results.

\begin{tabular}{cccc}
\hline Damaged story & $\begin{array}{c}\text { Evaluated stiffness } \\
(\mathbf{M P a})\end{array}$ & $\begin{array}{c}\text { Calculated by FEM } \\
(\mathbf{M P a})\end{array}$ & Percentage of error \\
\hline$\# 1$ & 13.96 & 13.96 & $\approx 0$ \\
$\# 6$ & 5.17 & 5.11 & 1.2 \\
\hline
\end{tabular}
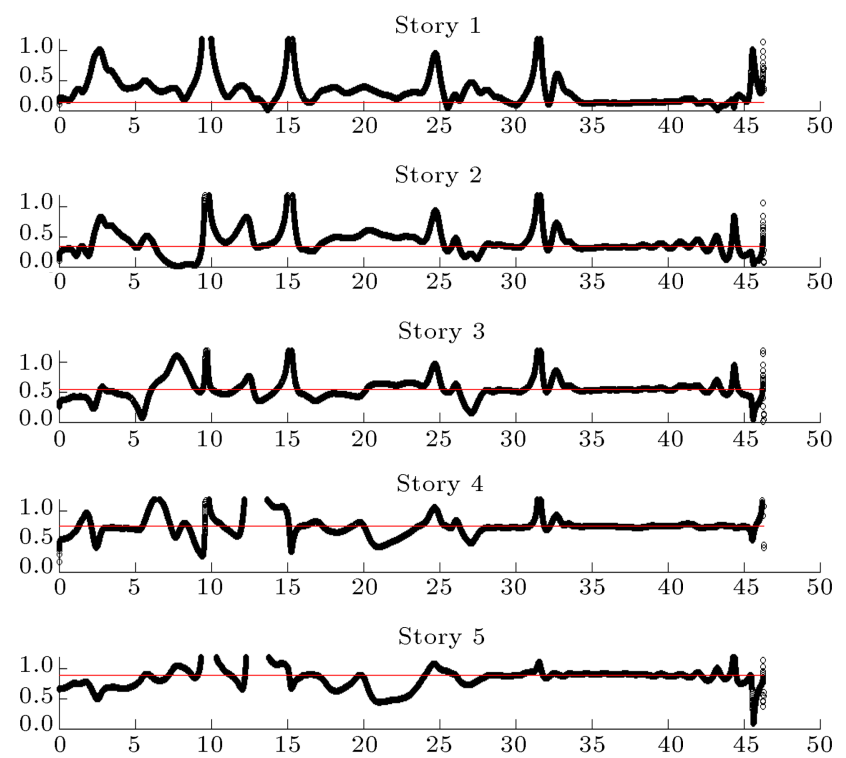

Story 6

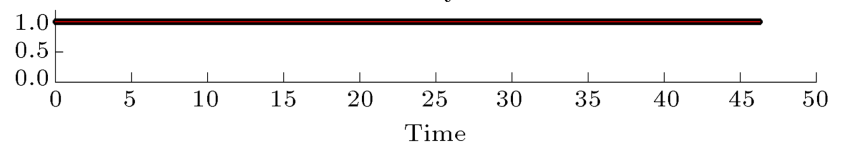

Figure 13. Normalized amplitude of IMFs 7 for each story relative to story 6 .

Consequently, the results obtained after using EEMD and Hilbert transform as well as the results obtained after employing ANNs are listed in Tables 5 and 6 , respectively.

Table 5 shows that there is not any significant error between the first-mode frequency calculated by FEM model and EEMD and Hilbert. In addition, Table 6 indicates that the proposed method detects the location of damages correctly and estimates the stiffness changes, too.
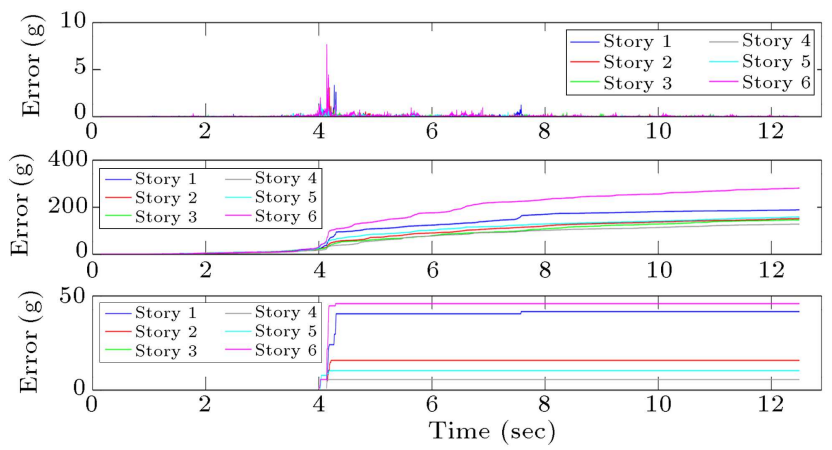

Figure 14. The difference between structure response and neural network prediction.

Figure 15 emphasizes the aforementioned fact, meaning that it is not necessary to precisely evaluate the first-mode frequency. In other words, this figure depicts that changing the first-mode frequency in the input how changes the final results in stiffness of the first and sixth stories.

\section{Conclusion remarks}

In this study, EMD is supplanted by EEMD in the process of HT. Even though these methods closely resemble each other, EEMD brings about more appropriate IMFs employed to assess the first-mode frequency and mode shape. To put it differently, using EEMD rather than EMD in HHT significantly improves its performance. By doing so, IMFs are more uniform, paving the way for assessing the first-mode shape. Afterward, an ANN was applied to predict story acceleration based on the acceleration response of the structure during the previous moments. ANNs imitate the behavior of the frame in the elastic range precisely; therefore, any congruency between the predicted and 

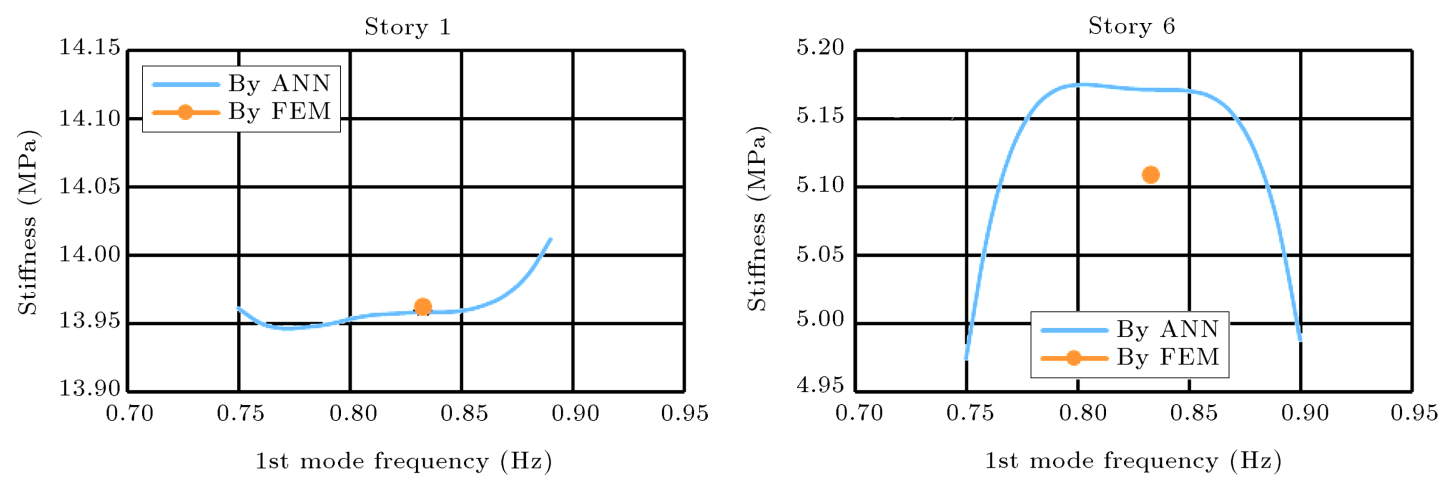

Figure 15. Sensitivity of the proposed algorithm to the first mode dominant frequency.

measured accelerations provides the onset of damage. Then, another ANN method was applied to estimate stiffness matrix. Though the first-mode shape and frequency are calculated in advance, it is essentially required for an inverse problem to be solved in order to find stiffness matrix, and this task is done by the second ANN. In other words, these two ANN methods were implemented to predict the location and measure the severity of damage, respectively. This algorithm was implemented on two moment-resisting steel frames, and the results were found acceptable. In fact, a novel technique based on the combination of a timeseries method and ANNs was applied, which not only overcomes the limitations of time-frequency methods for damage detection of nonlinear structures, but also provides an online monitoring method, which is essential in taking preventive measures. Furthermore, this approach is not sensitive to noise in input or output data, and even $10 \%$ error in assessing the first-mode frequency is negligible.

\section{References}

1. Adeli, H. "Neural networks in civil engineering: 19892000", Comput. Civ. Infrastruct. Eng., 16(2), pp. 126142 (2001).

2. Chang, P.C. and Liu, S.C. "Recent research in nondestructive evaluation of civil infrastructures", J. Mater. Civ. Eng., 15(3), pp. 298-304 (2003).

3. Fan, W. and Qiao, P. "Vibration-based damage identification methods: a review and comparative study", Struct. Heal. Monit., 10(1), pp. 83-111 (2011).

4. Lynch, J.P. and Loh, K.J. "A summary review of wireless sensors and sensor networks for structural health monitoring", Shock Vib. Dig., 38(2), pp. 91-130 (2006).

5. Doebling, S.W., Farrar, C.R., and Prime, M.B. "A summary review of vibration-based damage identification methods", Shock Vib. Dig., 30(2), pp. 91-105 (1998).

6. Yan, Y.J., Cheng, L., Wu, Z.Y., and Yam, L.H. "Development in vibration-based structural damage detection technique", Mech. Syst. Signal Process., 21(5), pp. 2198-2211 (2007).

7. Montalvao, D., Maia, N.M.M., and Ribeiro, A.M.R. "A review of vibration-based structural health monitoring with special emphasis on composite materials", Shock Vib. Dig., 38(4), pp. 295-326 (2006).

8. Goyal, D. and Pabla, B.S. "The vibration monitoring methods and signal processing techniques for structural health monitoring: A review", Arch. Comput. Methods Eng., 23(4), pp. 585-594 (2016). https://link.springer.com/article/10.1007/s11831-0159145-0

9. Chang, P.C., Flatau, A., and Liu, S.C. "Review paper: Health monitoring of civil infrastructure", Struct. Heal. Monit., 2(3), pp. 257-267 (2003).

10. Qarib, H. and Adeli, H. "Recent advances in health monitoring of civil structures", Sci. Iran., 21(6), pp. 1733-1742 (2014).

11. Loh, C.-H., Wu, T.-C., and Huang, N.E. "Application of the empirical mode decomposition-Hilbert spectrum method to identify near-fault ground-motion characteristics and structural responses", Bull. Seismol. Soc. Am., 91(5), pp. 1339-1357 (2001).

12. Yang, J., Lei, Y., Lin, S., and Huang, N. "HilbertHuang based approach for structural damage detection", J. Eng. Mech., 130, pp. 85-95 (January 2004).

13. Zhang, R.R., Ma, S., Safak, E., and Hartzell, S. "Hilbert-Huang transform analysis of dynamic and earthquake motion recordings", J. Eng. Mech., 129(8), pp. 861-875 (2003).

14. Hou, Z., Noori, M., and Amand, R. St. "Wavelet-based approach for structural damage detection", J. Eng. Mech., 126(7), pp. 677-683 (2000).

15. Li, H., Zhang, Y., and Zheng, H. "Hilbert-Huang transform and marginal spectrum for detection and diagnosis of localized defects in roller bearings", $J$. Mech. Sci. Technol., 23(2), pp. 291-301 (2009).

16. Amini Tehrani, H., Bakhshi, A., and Akhavat, M. "An effective approach for structural damage localization in flexural members based on generalized S-transform", Sci. Iran. (In Press). http://scientiairanica.sharif.edu/article_20019.html 
17. Huang, N.E., Shen, Z., Long, S.R., Wu, M.C., Shih, H.H., Zheng, Q., Yen, N.-C., Tung, C.C., and Liu, H.H. "The empirical mode decomposition and the hilbert spectrum for nonlinear and non-stationary time series analysis", Proc. R. Soc. London A Math. Phys. Eng. Sci., 454(1971), pp. 903-995 (1998).

18. Huang, N., Long, S., and Shen, Z. "The mechanism for frequency downshift in nonlinear wave evolution", Adv. Appl. Mech., 32, pp. 59-117 (1996). https://www.sciencedirect.com/science/article/pii/S0 065215608700760

19. Chen, B., Zhao, S., and Li, P. "Application of HilbertHuang transform in structural health monitoring: A state-of-the-art review", Math. Probl. Eng., 2014(7), pp. 1-22 (2014).

20. Huang, N.E., Shen, Z., and Long, S.R. "A new view of nonlinear water waves: The hilbert spectrum1", Annu. Rev. Fluid Mech., 31(1), pp. 417-457 (1999).

21. Vincent, H.T., Hu, S.L.J., and Hou, Z. "Damage detection using empirical mode decomposition method and a comparison with wavelet analysis", in $2 n d$ International Workshop on Structural Health Monitoring, Stanford University, pp. 891-900 (1999).

22. Wu, Z. and Huang, N.E. "A study of the characteristics of white noise", R. Soc., 460, pp. 1597-1611 (2004).

23. Wu, Z. and Huang, N.E., Ensemble Empirical Mode Decomposition, World Sci. Publ., 1(1), pp. 1-41 (2009).

24. Aied, H., González, A., and Cantero, D. "Identification of sudden stiffness changes in the acceleration response of a bridge to moving loads using ensemble empirical mode decomposition", Mech. Syst. Signal Process., 6667, pp. 314-338 (2016).

25. Moser, P. and Moaveni, B. "Environmental effects on the identified natural frequencies of the Dowling Hall Footbridge", Mech. Syst. Signal Process., 25(7), pp. 2336-2357 (2011).

26. Nagarajaiah, S. and Basu, B. "Output only modal identification and structural damage detection using time frequency \& wavelet techniques", Earthq. Eng. Eng. Vib., 8(4), pp. 583-605 (2009).

27. Bishop, C.M., Neural Networks for Pattern Recognition, Oxford University Press, Inc. (1995).

28. Suresh, S., Omkar, S.N., Ganguli, R., and Mani, V. "Identification of crack location and depth in a cantilever beam using a modular neural network approach", Smart Mater. Struct., 13(4), pp. 907-915 (2004).

29. Fang, X., Luo, H., and Tang, J. "Structural damage detection using neural network with learning rate improvement", Comput. Struct., 83(25-26), pp. 21502161 (2005).

30. Xu, B., Wu, Z., Chen, G., and Yokoyama, K. "Direct identification of structural parameters from dynamic responses with neural networks", Eng. Appl. Artif. Intell., 17, pp. 931-943 (2004).
31. Saadat, S., Buckner, G.D., Furukawa, T., and Noori, M.N. "An intelligent parameter varying (IPV) approach for non-linear system identification of base excited structures", Int. J. Non. Linear. Mech., 39(6), pp. 993-1004 (2004).

32. Bandara, R.P., Chan, T.H.T., and Thambiratnam, D.P. "Structural damage detection method using frequency response functions", Struct. Heal. Monit., 13(4), pp. 418-429 (Feb. 2014).

33. Rafiei, M.H. and Adeli, H. "A novel unsupervised deep learning model for global and local health condition assessment of structures", Eng. Struct., 156, pp. 598607 (2018).

34. Entezami, A., Shariatmadar, H., and Karamodin, A. "An improvement on feature extraction via time series modeling for structural health monitoring based on unsupervised learning methods", Sci. Iran. (In Press). http://scientiairanica.sharif.edu/article_20641_0.html

35. Amezquita-Sanchez, J.P. and Adeli, H. "Feature extraction and classification techniques for health monitoring of structures", Sci. Iran. Trans. A, Civ. Eng., 22(6), p. 1931 (2015).

36. Huang, N.E. and Shen, S.S., Hilbert-Huang Transform and Its Applications, (World Scientific, 5 (2005).

37. Huang, N.E., Computing Instantaneous Frequency by Normalizing Hilbert Transform, Patent 6901353, U.S. Patent and Trademark Off., Washington, D.C. (2005).

38. Bahar, O. and Ramezani, S. "Enhanced Hilbert-Huang transform and its application to modal identification", Struct. Des. Tall Spec. Build., 23(4), pp. 239-253 (2014).

39. Wang, T., Zhang, M., Yu, Q., and Zhang, H. "Comparing the applications of EMD and EEMD on timefrequency analysis of seismic signal", J. Appl. Geophys., 83, pp. 29-34 (2012).

40. Jiang, S., Wu, S., and Dong, L. "A time-domain structural damage detection method based on improved multiparticle swarm coevolution optimization algorithm", Math. Probl. Eng., 2014, p. 11 (2014).

41. Friswell, M.I., Penny, J.E.T., and Garvey, S.D. "A combined genetic and eigensensitivity algorithm for the location of damage in structures", Comput. Struct., 69(5), pp. 547-556 (1998).

\section{Biographies}

Sayyed Mohsen Vazirizade is a $\mathrm{PhD}$ candidate at University of Arizona and graduated from Sharif University in 2015. His focus of research includes reliability engineering and machine learning and application of uncertainty and AI in engineering structures.

Ali Bakhshi is an Associate Professor at Civil Engineering Department at Sharif University of Technology. He obtained his PhD degree from Hiroshima University of Technology, Hiroshima, Japan. Furthermore, he 
is the Director of Earthquake Simulation National Lab and the Head of Earthquake Engineering Research Center at Sharif University. His main research interests include earthquake modeling and random vibration.

Omid Bahar is an Assistant Professor at International Institute of Earthquake Engineering and Seismology
(IIEES), Tehran, Iran. He earned his $\mathrm{PhD}$ degree in Structural Engineering from Shiraz University, Shiraz, Iran. He spent a short while as a visiting scholar in Professor Kitagawa's Lab. in Hiroshima University during his $\mathrm{PhD}$ research program, he worked on the performance of active structural control during strong ground motions. His main research interests include signal processing and structural health monitoring. 